\section{Bright Coherent Ultrahigh Harmonics in the keV X-ray Regime from Mid-Infrared Femtosecond Lasers}

Tenio Popmintchev,${ }^{1 *}$ Ming-Chang Chen, ${ }^{1}$ Dimitar Popmintchev, ${ }^{1}$ Paul Arpin, ${ }^{1}$ Susannah Brown, ${ }^{1}$ Skirmantas Ališauskas, ${ }^{2}$ Giedrius Andriukaitis, ${ }^{2}$ Tadas Balčiunas, ${ }^{2}$ Oliver D. Mücke, ${ }^{2}$ Audrius Pugzlys, ${ }^{2}$ Andrius Baltuška, ${ }^{2}$ Bonggu Shim, ${ }^{3}$ Samuel E. Schrauth, ${ }^{3}$ Alexander Gaeta, ${ }^{3}$ Carlos Hernández-García, ${ }^{4}$ Luis Plaja, ${ }^{4}$ Andreas Becker, ${ }^{1}$ Agnieszka Jaron-Becker, ${ }^{1}$ Margaret M. Murnane, ${ }^{1}$ Henry C. Kapteyn ${ }^{1}$

High-harmonic generation (HHG) traditionally combines $~ 100$ near-infrared laser photons to generate bright, phase-matched, extreme ultraviolet beams when the emission from many atoms adds constructively. Here, we show that by guiding a mid-infrared femtosecond laser in a high-pressure gas, ultrahigh harmonics can be generated, up to orders greater than 5000 , that emerge as a bright supercontinuum that spans the entire electromagnetic spectrum from the ultraviolet to more than 1.6 kilo-electron volts, allowing, in principle, the generation of pulses as short as 2.5 attoseconds. The multiatmosphere gas pressures required for bright, phase-matched emission also support laser beam self-confinement, further enhancing the $x$-ray yield. Finally, the $x$-ray beam exhibits high spatial coherence, even though at high gas density the recolliding electrons responsible for HHG encounter other atoms during the emission process.

$\mathrm{T}$ he unique ability of $\mathrm{x}$-rays to capture structure and dynamics at the nanoscale has spurred the development of large-scale $\mathrm{x}$-ray free-electron lasers based on accelerator physics, as well as high-harmonic generation (HHG) techniques in the x-ray region that employ tabletop femtosecond lasers. The HHG process represents nonlinear optics at an extreme, enabling femtosecond-to-attosecond duration pulses with full spatial coherence (1-O), which make it possible to track the dynamics of electrons in atoms, molecules, and materials (7-12). X-rays can probe the oxidation or spin state in molecules and materials with element specificity, because the position of the characteristic $\mathrm{x}$-ray absorption edges of individual elements is sensitive to the local environment and structure. Ultrashort x-ray pulses can capture the coupled motions of charges, spins, atoms, and phonons by monitoring changes in absorption or reflection that occur near these edges as a material or molecule changes state or shape. However, many inner-shell absorption edges in advanced correlated-electron, magnetic, and catalytic materials $(\mathrm{Fe}, \mathrm{Co}, \mathrm{Ni}, \mathrm{Cu})$ lie at photon energies nearing 1 kilo-electron volt $(\mathrm{keV})$ (13-15). In contrast, most applications that use
HHG light have been limited to the extreme ultraviolet (EUV) region of the spectrum $(<150 \mathrm{eV})$, where efficient frequency upconversion is possible with the use of widely available Ti:sapphire lasers operating at a $0.8-\mu \mathrm{m}$ wavelength. We therefore sought to extend bright HHG to a higher-energy soft x-ray region.

High-harmonic generation is a universal response of atoms and molecules in strong femtosecond laser fields $(16,17)$. In a simple analogy, HHG represents the coherent version of the Röntgen $\mathrm{x}$-ray tube: Instead of boiling electrons off a hot filament, accelerating them in an electric field, and generating incoherent $\mathrm{x}$-rays when the highenergy electrons strike a target, HHG begins with tunnel ionization of an atom in a strong laser field. The portion of the electron wave function that escapes the atom is accelerated by the laser electric field and, when driven back to its parent ion by the laser, can coherently convert its kinetic energy into a high-harmonic photon. The highestenergy HHG photon emitted is given by the microscopic single-atom cutoff rule: $h v_{\mathrm{SA}}$ cutoff $=$ $I_{\mathrm{p}}+3.17 U_{\mathrm{p}}$, where $h$ is Planck's constant, $v$ is the frequency, $I_{\mathrm{p}}$ is the ionization potential of the gas, and $U_{\mathrm{p}} \propto I_{\mathrm{L}} \lambda_{\mathrm{L}}{ }^{2}$ is the quiver energy of the liberated electron in a laser field of intensity $I_{\mathrm{L}}$ and wavelength $\lambda_{\mathrm{L}}$

Generating bright, fully coherent HHG beams requires macroscopic phase matching $(18)$, wherein

${ }^{1}$ JILA, University of Colorado at Boulder, Boulder, CO 80309, USA. ${ }^{2}$ Photonics Institute, Vienna University of Technology, Vienna A-1040, Austria. ${ }^{3}$ School of Applied and Engineering Physics, Cornell University, Ithaca, NY 14853, USA. ${ }^{4}$ Grupo de Investigación en Optica Extrema, Universidad de Salamanca, Salamanca E37008, Spain.

*To whom correspondence should be addressed. E-mail: popmintchev@jila.colorado.edu
A
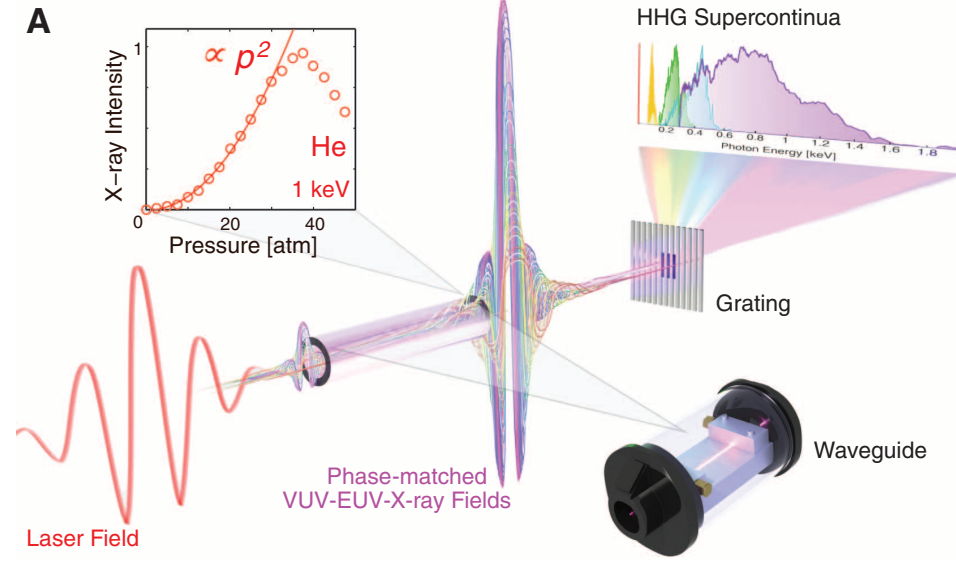

Fig. 1. (A) Schematic illustration of the coherent kilo-electron volt x-ray supercontinua emitted when a mid-IR laser pulse is focused into a high-pressure gas-filled waveguide. The experimental phase-matched harmonic signal grows quadratically with pressure, demonstrating excellent phase-matched coherent buildup with increasing pressure $p$. (B) Experimental HHG spectra emitted under full phasematching conditions as a function of driving-laser wavelength (yellow, $0.8 \mu \mathrm{m}$; green, $1.3 \mu \mathrm{m}$; blue, $2 \mu \mathrm{m}$; purple, $3.9 \mu \mathrm{m}$ ). (Inset) Fourier transform-limited pulse duration of 2.5 as. (C) Calculated spectrum and temporal structure of one of the phase-matched HHG bursts driven by a six-cycle FWHM 3.9- $\mu \mathrm{m}$ pulse at a laser intensity of $I_{\mathrm{L}}=3.3 \times 10^{14} \mathrm{~W} / \mathrm{cm}^{2}$.
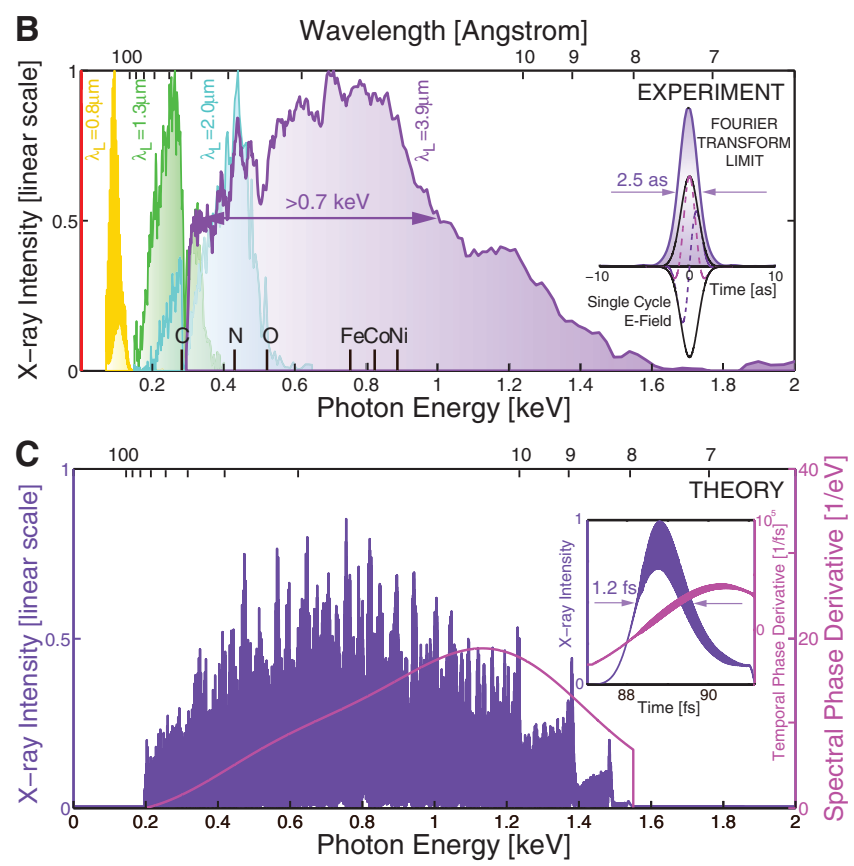
the laser and high-order nonlinear polarization propagate in phase (at the speed of light) throughout a medium to ensure that the HHG light emitted from many atoms adds coherently $(1,19,20)$. Phase matching is achieved by balancing the neutral gas and free-electron plasma dispersion experienced by the laser and is only possible up to some critical ionization level that depends on the gas species and laser wavelength (fig. S1). Any geometric contributions to the laser propagation must also be considered (see supplementary text). Because ionization increases with laser intensity, the critical ionization limits the highest photon energy for which phase matching can be implemented. Recent work explored the wavelength dependence of the HHG yield (21-24), which scales as $h v_{\mathrm{PM} \text { cutoff }} \propto \lambda_{\mathrm{L}}{ }^{1.7}$ under phase-matched conditions (25-27). Using 2- $\mu \mathrm{m}$ lasers $(0.62-\mathrm{eV}$ photons) to drive HHG, bright harmonics extend to $>0.5 \mathrm{keV}$ (26), demonstrating phase matching of a $>800$-order nonlinear process (note that only odd-order harmonics are emitted to conserve angular momentum).

In this work, bright high-harmonic x-ray supercontinua with photon energies spanning from the EUV to $1.6 \mathrm{keV}(<7.7 \AA)$ are generated by focusing 3.9- $\mu \mathrm{m}$ wavelength pulses from a tabletop femtosecond laser into a waveguide filled with He gas (see Fig. 1). This represents an extreme $>5000$-order nonlinear process while also demonstrating fully phase-matched frequency upconversion. The multiatmosphere pressures necessary for efficient $\mathrm{x}$-ray generation also support laser beam self-confinement, enhancing the $\mathrm{x}$-ray yield by another order of magnitude. We observe coherent, laserlike x-ray beams, despite the fact that ultrahigh-harmonic generation occurs in a regime where the laser-driven electrons encounter many neighboring atoms before they re-encounter their parent ions. Our calculations indicate that the kilo-electron volt - bandwidth coherent supercontinuum has a well-behaved chirp that, when compensated, could support a single-x-ray-cycle 2.5-attosecond pulse duration. Finally, we show that in the kilo-electron volt region, a much higher-order nonlinear process is required for phase matching than is required for harmonic emission from a single atom.

In our experiment, six-cycle full width at half maximum (FWHM) (80-fs) 10-mJ pulses, centered at a wavelength of $3.9 \mu \mathrm{m}$, are generated at $20 \mathrm{~Hz}$ as the idler output of an optical parametric chirped-pulse amplification laser system $(28,29)$. $\mathrm{X}$-rays are generated by focusing the laser beam into a $200-\mu \mathrm{m}$ diameter, 5-cm-long, gas-filled hollow waveguide capable of sustaining pressures of up to $80 \mathrm{~atm}$ in a differentially pumped geometry. The HHG spectrum is then captured with the use of a soft $\mathrm{x}$-ray spectrometer and $\mathrm{x}$-ray chargecoupled device camera. Figure 1B shows the phase-matched HHG emission from $\mathrm{He}$, which extends to $>1.6 \mathrm{keV}(<7.7 \AA)$. The phase-matched HHG cutoff energy agrees well with numerical predictions plotted in Fig. 2A for 3.9- $\mu \mathrm{m}$ driving lasers; that is, $h v_{\mathrm{PM} \text { cutoff }} \propto \lambda_{\mathrm{L}}{ }^{1.7}(25-27)$. This bright $\mathrm{x}$-ray supercontinuum is ideal for $\mathrm{x}$-ray spectroscopy measurements, spanning multiple inner-shell absorption edges simultaneously (Fig. $1 \mathrm{~B}$ and fig. S2), as has already been demonstrated in the EUV region for HHG driven by multicycle $0.8-\mu \mathrm{m}$ lasers where a quasi-continuous $\mathrm{HHG}$ spectrum is emitted $(15,30,31)$.

The x-ray flux from He scales quadratically with pressure (number of emitters), as shown in Fig. 1A, reaching a maximum at very high gas pressures of $\sim 35 \mathrm{~atm}$, where both phase matching and laser beam self-confinement are optimized. At higher pressures, the x-ray flux decreases due to reabsorption of the generated harmonics by the high-pressure gas, as well as energy loss experienced by the laser when coupling into the waveguide. Microscopically, quantum diffusion leads to spreading of the electron wave packet, decreasing the recombination probability and, thus, the single-atom HHG yield (22-24), which scales with the laser wavelength as $\sim \lambda_{\mathrm{L}}{ }^{-6.5}$ under phase-
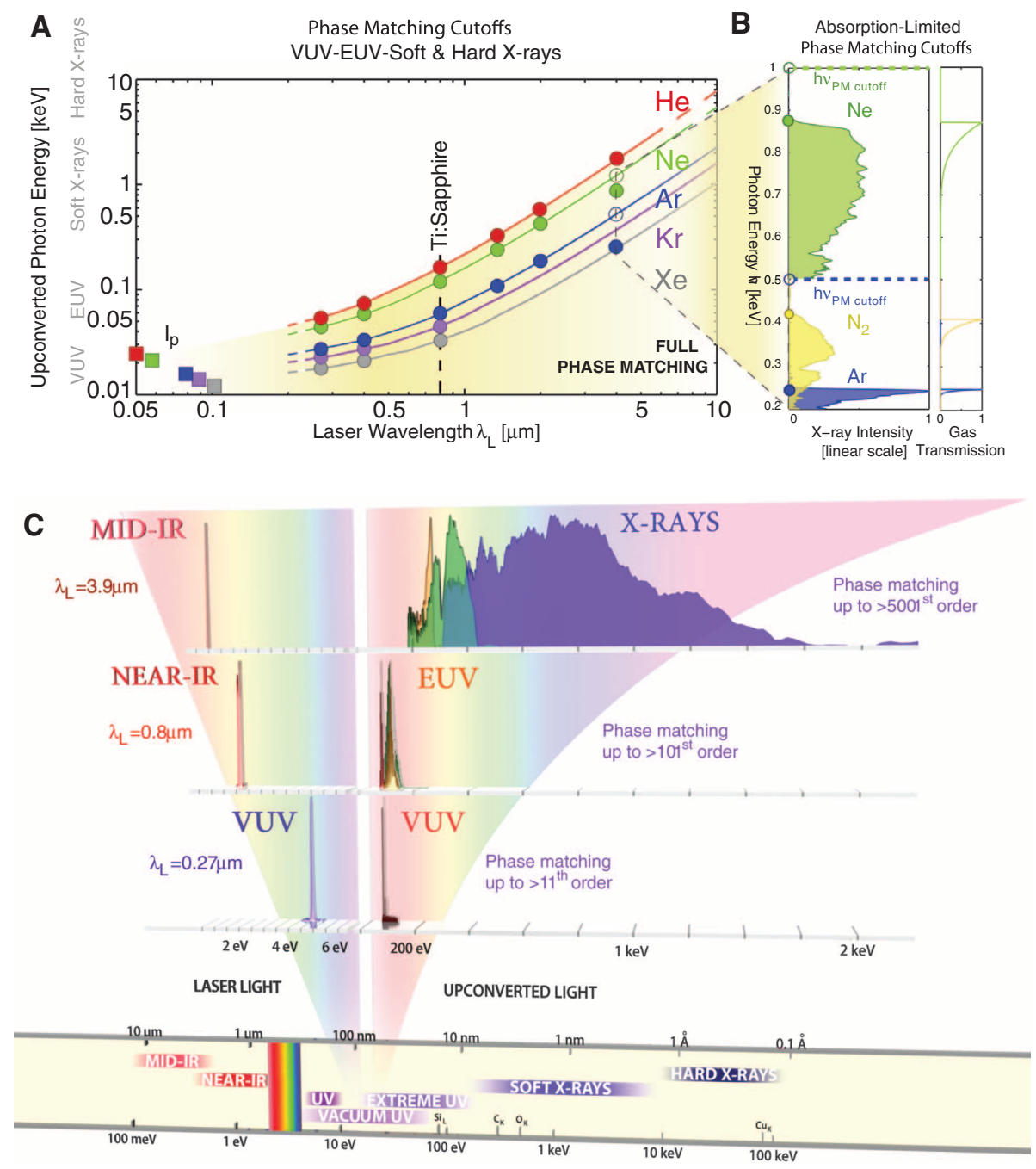

Fig. 2. (A) Predicted and observed HHG phase-matching cutoffs as a function of laser wavelength from the UV to the mid-IR. Solid circles show the observed cutoffs; open circles show the predicted cutoffs for Ar and $\mathrm{Ne}$ [which cannot be reached due to inner-shell absorption, as shown in (B)]. Solid squares on the left show the ionization potentials $\left(I_{\mathrm{p}}\right)$ of the different atoms. (C) Unified picture of optimal phase-matched high-harmonic upconversion, including microscopic and macroscopic effects. 
this regime (14). Thus, surprisingly, for macroscopic phase matching, the required harmonic order of $>5000$ is much higher than that required to generate the same photon energy from a single atom using a shorter laser wavelength.

We can now present a unified picture for phasematched high-harmonic upconversion, spanning the electromagnetic spectrum from the vacuum ultraviolet (VUV) to greater than kilo-electron volt x-ray photon energies, that includes both the microscopic and macroscopic physics. To validate theory, we tuned our driving laser to different wavelengths from the UV to the mid-infrared (mid-IR) and then implemented pressure-tuned phase matching to optimize the HHG flux at each laser wavelength with the optimal laser intensities dictated by the critical ionization of the medium (Fig. 2 and supplementary text). The required optimal pressures and interaction lengths evolve from $<0.1 \mathrm{~atm}$ and a few millimeters in the VUV region to tens of atmospheres and multicentimeter lengths in the $\mathrm{x}$-ray region. Figure 2, A and $\mathrm{C}$, shows the optimized phase-matched cutoffs and spectra for different driving-laser wavelengths. To efficiently generate high harmonics, the order of the nonlinearity must increase from $\sim 11$ in the VUV to $>5000$ in the kilo-electron volt region. This represents an extreme for both the order of a nonlinear process and phase matching. The bright phase-matched HHG spectra evolve from a single harmonic in the VUV into a broad x-ray supercontinuum spanning thousands of harmonics in the soft x-ray region. Phase matching shuts off in the VUV at energies near the ionization potential of the nonlinear gas medium, as the HHG and driving-laser wavelengths converge (see Fig. 2A). The phase-matched HHG conversion efficiencies reach $10^{-3}$ to $10^{-4}$ in the VUV region, compared with $10^{-5}$ in the EUV using $0.8-\mu \mathrm{m}$ lasers, and $10^{-6}$ to $10^{-7}$ in the $\mathrm{x}$-ray region. Moreover, in the VUV region, phase matching occurs at relatively high levels of ionization of tens of percent (fig. S1).

Remarkably, tunnel ionization of the atomic gas medium dominates in all phase-matching regimes. When driven by UV light, the effective potential (which is a superposition of the Coulomb and laser fields) oscillates rapidly, allowing a very short time interval for the electron to tunnel. However, the required laser intensity for $\mathrm{HHG}$ is extremely high $\left(>10^{15} \mathrm{~W} / \mathrm{cm}^{2}\right)$, so tunnel ionization is more probable than multiphoton ionization. For mid-IR laser wavelengths, the slowly oscillating effective Coulomb potential can be considered quasi-static. Therefore, although the laser intensity decreases to maintain phase matching, tunnel ionization is still more probable than multiphoton ionization. Because the physics of ionization does not change, we can use an analytical description of tunneling [the AmossovDelone-Krainov model (32)] to derive a generalized analytic HHG phase matching cutoff rule (Eq. 1), validated by comparison with experiment, as well as numerical and quantum theory (see supplementary text)

$$
h v_{\mathrm{PM} \text { cutoff }}=I_{\mathrm{p}}+\frac{\alpha I_{\mathrm{p}}^{3}}{\ln ^{2}\left\{\frac{\beta I_{\mathrm{p}} \tau_{\mathrm{L}}}{-\ln \left[1-\eta_{\mathrm{CR}}\left(\lambda_{\mathrm{L}}\right)\right]}\right\}} \lambda_{\mathrm{L}}^{2}
$$

Here, $\alpha$ and $\beta$ are constants that depend on the laser pulse shape and the state from which the electron is tunnel ionized, $\tau_{\mathrm{L}}$ is the laser pulse duration, and $\eta_{\mathrm{CR}}$ is the critical ionization. This analytical expression gives some physical insight into phase matching of the HHG upconversion process. The small deviation of $\lambda_{\mathrm{L}}{ }^{(1.5-1.7)}$ from the $\lambda_{\mathrm{L}}{ }^{2}$ scaling of the ponderomotive energy incorporates the proper scaling of the laser intensity and arises from the scaling of $\eta_{\mathrm{CR}}$, which decreases by four orders of magnitude from the UV to mid-IR driving-laser wavelengths. Short, few-cycle laser pulses make it possible to generate higher-energy photons before the critical ionization level is exceeded. However, this approach yields diminishing returns for pulses shorter than 5 to 10 cycles and leads to only modest enhancements in HHG flux and phasematching cutoff. The most substantial HHG enhancement (by orders of magnitude) arises when the right combination of laser wavelength, gas pressure-length product, and laser intensity is used.

Likewise, in contrast to conventional wisdom (see supplementary text), helium is generally the best atomic medium for harmonic generation due to the absence of inner-shell absorption (25). The absorption limit for HHG emission can be clearly seen in Fig. 2B, which shows a plot of the phasematched $\mathrm{HHG}$ emission from $\mathrm{Ar}, \mathrm{N}_{2}$, and $\mathrm{Ne}$ when driven by $3.9-\mu \mathrm{m}$ light. There is a sharp drop-off in signal at the inner-shell absorption edges at $0.25(\mathrm{Ar}), 0.41\left(\mathrm{~N}_{2}\right)$, and $0.87 \mathrm{keV}(\mathrm{Ne})$; therefore, the true phase-matching cutoff cannot be observed: Without absorption, the phasematching limits would be $\sim 0.5\left(\mathrm{Ar}, \mathrm{N}_{2}\right)$ and $\sim 1 \mathrm{keV}(\mathrm{Ne})$.

Generating bright kilo-electron volt harmonics from atoms driven by mid-IR femtosecond lasers takes advantage of a noteworthy convergence of favorable physics. First, the very high gas density required puts these experiments in a regime of HHG from nonisolated emitters: Spread of the ionized electron quantum wave packet over its few-femtosecond free trajectory means that the electron will encounter many neighboring atoms.

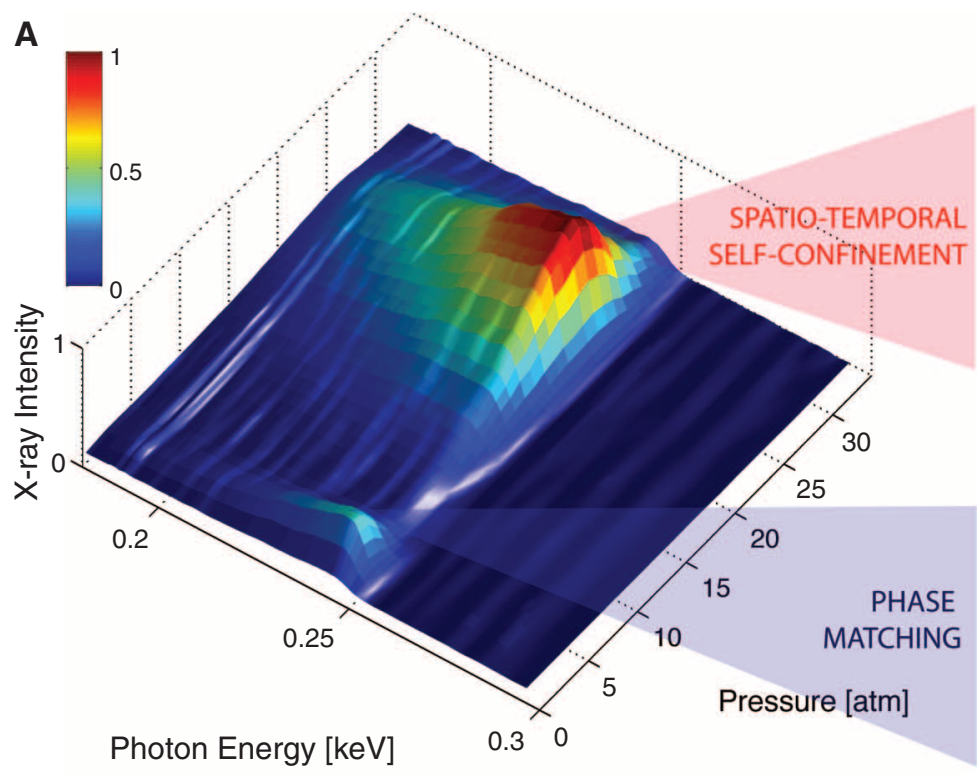

Fig. 3. (A) Measured HHG yield in Ar as a function of pressure and photon energy, showing two peaks: one at 3-atm pressure due to pressure-tuned phase matching and a second at 26-atm pressure due to the additional presence of laser-beam spatio-temporal self-confinement. (B) Experimental HHG beam profiles and calculated laser-beam profiles after a propagation distance of $3.8 \mathrm{~cm}$ in the waveguide. arb. u., arbitrary units.
B X-RAY PROFILES X-ray wavelength [arb. u.]
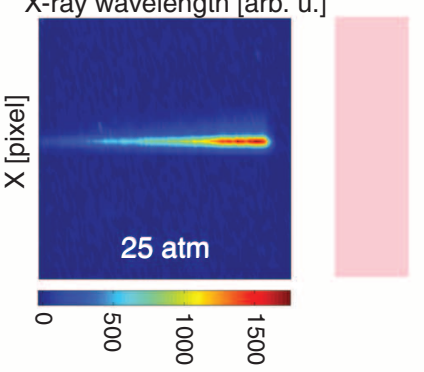

X-ray wavelength [arb. u.]
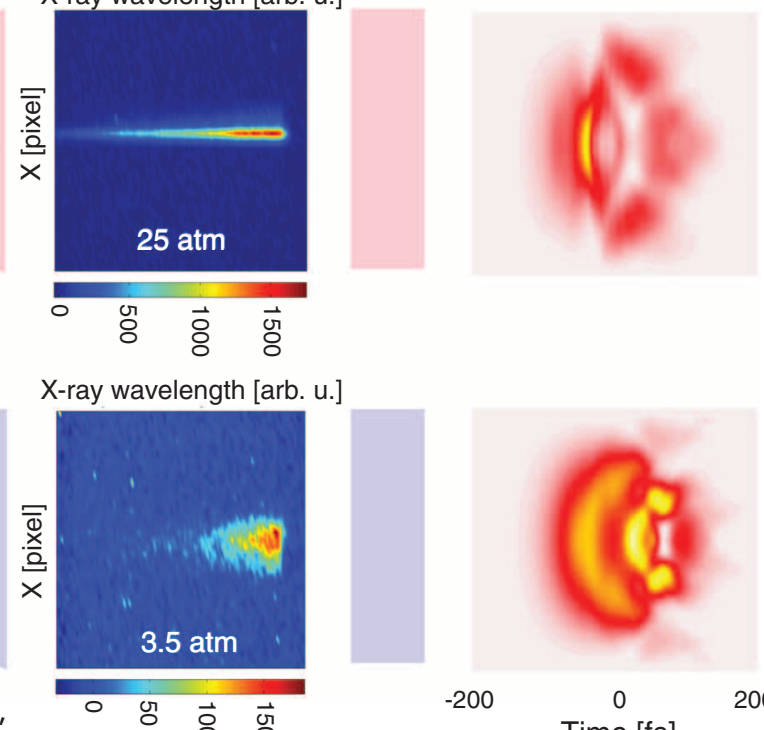

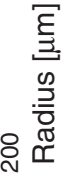

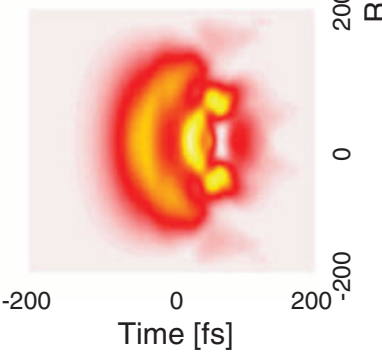


This contrasts with emission from dilute, isolated atoms for UV or EUV harmonic generation. As shown in fig. S1, for kilo-electron volt harmonics, the electron wave function in the continuum extends to $\sim 500 \AA$, whereas the separation between the He atoms is $\sim 15 \AA$ at 10 -atm pressure. However, the ionization levels are low at $\sim 0.03 \%$. For VUV/EUV harmonics, the electron typically extends $\sim 2$ to $20 \AA$ between ionization and recollision, whereas the separation between atoms is $\sim 70 \AA$ at $\sim 0.1 \mathrm{~atm}$ pressure, and phase matching occurs at $\sim 10 \%$ ionization levels. Thus, $\mathrm{HHG}$ driven by mid-IR pulses liberates 0.001 as much of the electron wave function into the continuum compared with visible driving lasers, though it is spread over a 100-times-larger distance. Fortunately, our experimental results indicate that rescattering of this large and diffuse recolliding electron wave packet from other atoms seems not to adversely influence the coherence of the emission, likely because the medium is weakly ionized. Evidence for this includes the well-formed, spatially coherent X-ray beams (Fig. 3B and Fig. 4) and the remarkable quadratic growth (Fig. 1A) that continues from $0.2 \mathrm{~atm}$ (when the rescattering electron wave packet can begin to encounter neighboring atoms) to more than two-orders-ofmagnitude-higher pressure.

In a second extremely favorable convergence of extreme nonlinear optics, the multiatmosphere gas pressures required for phase-matched $\mathrm{x}$-ray generation also overlap with the parameter range where laser beam self-confinement is possible. Figure $3 \mathrm{~A}$ shows a plot of the experimental x-ray emission from Ar driven by 3.9- $\mu \mathrm{m}$ lasers. The predicted phase-matching pressure is $\sim 3 \mathrm{~atm}$, and we indeed observe a peak in x-ray emission at that pressure. However, as the pressure is further increased, the $\mathrm{x}$-ray yield first decreases and then increases quadratically, exhibiting a large enhancement at a pressure of $26 \mathrm{~atm}$ (about a factor of 10 when integrated over all soft x-ray HHG). The measured $\mathrm{x}$-ray beam profile also dramatically narrows as the gas pressure increases (Fig. 3B), indicative of self-confinement of the driving laser. Essentially, the x-ray HHG beam, imaged at the exit of the fiber, shrinks to less than one-third of its former diameter, whereas the x-ray signal increases tenfold (integrated over all orders) at pressures seven times greater than those required for phase matching.

To explore theoretically how macroscopic nonlinear effects augment HHG phase matching, we numerically simulated nonlinear pulse propagation in a hollow waveguide filled with high-pressure gas by extending and expanding previous simulations to longer wavelengths $(33,34)$. Our simulations show that as the gas pressure increases beyond that required for phase matching, the peak laser intensity is stabilized (figs. S3 and S4). We also observe strong spatio-temporal compression and localization of the driving laser during self-confinement due to the Kerr effect and plasma generation, which also enhances the HHG yield. Figure 3B plots the calculated beam pro- files at the phase-matching (3.5 atm) and higher pressures $(26 \mathrm{~atm})$. A stable self-confined beam forms at the higher gas pressures and persists for centimeter distances. As discussed in the supplementary text, we can experimentally and theoretically observe that self-confinement also enhances phase matching in other gases, such as $\mathrm{He}$ (fig. S4) and molecular $\mathrm{N}_{2}$.

When phase matched, the spatial quality of the $\mathrm{x}$-ray beam is excellent. Figure 4 shows the $\mathrm{x}$-ray beam and the Young's double-slit diffraction patterns taken by illuminating $5-\mu \mathrm{m}$ slits (separated by $10 \mu \mathrm{m}$ ) with an x-ray supercontinuum generated in $\mathrm{He}$ and $\mathrm{Ne}$, spanning 7.7 to $43 \AA$ and 14 to $43 \AA$, respectively. There is excellent agreement between the experimentally observed and theoretically predicted diffraction patterns. A plot of the expected diffraction pattern from incoherent $\mathrm{X}$-ray illumination is also shown in Fig. 4, B and C, for the same experimental geometry, proving that the high fringe visibility is not due to the small pinhole size but rather to the high spatial coherence of the x-ray beam itself. This measurement is extremely challenging at short wavelengths: Very small slit widths are required so that the light from each slit diffracts sufficiently to ensure overlap and interference at the detector (3.5 $\mathrm{m}$ away from the slits). Thus, the throughput is very small. This spatial coherence measurement clearly demonstrates that coherent diffractive imaging will be possible with near wavelength spatial resolution, as has been achieved using HHG beams and synchrotron sources in the EUV and soft x-ray regions $(35,36)$.

To predict the temporal properties of the $\mathrm{HHG}$ radiation, we theoretically analyzed HHG driven by one- and six-cycle FWHM mid-IR laser pulses, with peak intensities of 4.1 and $3.3 \times 10^{14} \mathrm{~W} / \mathrm{cm}^{2}$, respectively, from single atoms and also in a phase-matched regime. Our calculations, based on the strong field approximation and discrete dipole approach (37), confirm the femtosecond time scale of the x-ray bursts from a single atom and also after propagation (see supplementary text). Our calculated phase-matched HHG spectra agree well with those measured experimentally (Fig. 1, $\mathrm{B}$ and $\mathrm{C}$ ) and show that the HHG chirp is well behaved (Fig. 1C and fig. S5) over the near- kilo-electron volt bandwidth that, when compressed, is sufficient to support a single-cycle, 2.5 -as pulse in the Fourier limit. For 3.9- $\mu \mathrm{m}$ driving lasers in the single-atom case, contributions from the short and long trajectories lead to a parabolic chirp, whereas after propagation, the phasematched short trajectory contribution leads to a positive, quasi-linear chirp. The current limit of theory allows us to simulate HHG propagation over 20- $\mu \mathrm{m}$ distances at high pressures and predicts that the uncompressed HHG temporal emission consists of a series of $\sim$ three intense bursts of 1 - to 3 -fs duration, due to the very long 13 -fs period of the multicycle 3.9- $\mu \mathrm{m}$ driving laser field (Fig. 1C and fig. S5). However, for longer propagation distances, bright HHG emission in the form of a single isolated $\mathrm{x}$-ray burst is expected. This is because phase matching is transient and favors $\mathrm{x}$-ray emission from a single halfcycle of the laser pulse where the phase matching is optimal. This has been verified experimentally in the EUV, even without stabilizing the carrier wave with respect to the pulse envelope $(25,38)$. Interestingly, this work and past work predict that the HHG bursts are chirped, where the amount of chirp scales inversely with laser wavelength for a given spectral bandwidth (22). However, as shown in Fig. 1, the duration of each HHG burst still spans femtosecond durations (for example, 1000 times longer than their transform limit) due to the increased phase-matched HHG bandwidth, which scales almost as the square of the laser wavelength.

Experimental verification of these predictions will require the development of characterization methods that can sample ultrabroad bandwidth $\mathrm{x}$-ray waveforms at different photon energies. This challenge is illustrated in Fig. 1B, where the narrow dip at $0.54 \mathrm{keV}$ corresponds to oxygen $\mathrm{K}$-edge absorption. It is not clear that any atomic or molecular system can interact with a kiloelectron volt bandwidth, because processes such as photoionization involve significantly slower time scales. However, the chirped x-ray supercontinua already represent a promising multipleatomic site probe with subfemtosecond time resolution, analogous to the chirped white light (visible) continua used to probe many absorption
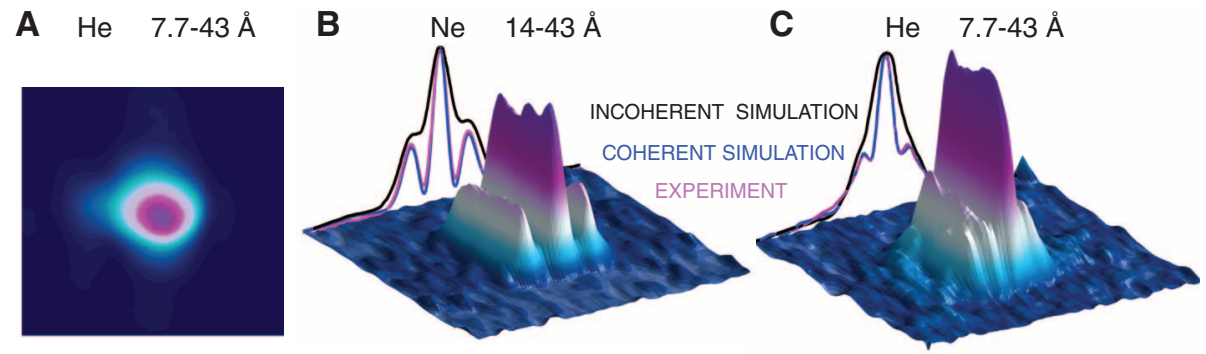

Fig. 4. (A) X-ray experimental beam profile. (B and C) Young's double-slit diffraction patterns taken by illuminating $5-\mu \mathrm{m}$ slits, separated by $10 \mu \mathrm{m}$, with the beam shown in $(\mathrm{A})$. There is excellent agreement between the experimentally observed (purple line) and theoretically predicted (blue line) diffraction patterns. The broad bandwidth and very low divergence of the HHG beams limit the number of fringes observed. The expected diffraction, assuming incoherent illumination, is also given for comparison (black line), illustrating the high spatial coherence of the kilo-electron volt HHG source. 
features simultaneously, perfectly synchronized to the driving laser. Given our current experimental and theoretical findings, it may be possible to extend HHG to hard $\mathrm{x}$-ray wavelengths and broader zeptosecond bandwidths.

\section{References and Notes}

1. A. Rundquist et al., Science 280, 1412 (1998).

2. R. A. Bartels et al., Science 297, 376 (2002).

3. M. Hentschel et al., Nature 414, 509 (2001).

4. D. G. Lee, ]. J. Park, J. H. Sung, C. H. Nam, Opt. Lett. 28, 480 (2003).

5. P. B. Corkum, N. H. Burnett, M. Y. Ivanov, Opt. Lett. 19, 1870 (1994).

6. I. P. Christov, M. M. Murnane, H. C. Kapteyn, Phys. Rev. Lett. 78, 1251 (1997).

7. R. Haight, Surf. Sci. Rep. 21, 275 (1995)

8. M. Bauer et al., Phys. Rev. Lett. 87, 025501 (2001).

9. L. Miaja-Avila et al., Phys. Rev. Lett. 101, 046101 (2008).

10. S. A. Aseyev, Y. Ni, L. ]. Frasinski, H. G. Muller, M. J. J. Vrakking, Phys. Rev. Lett. 91, 223902 (2003)

11. W. Li et al., Proc. Natl. Acad. Sci. U.S.A. 107, 20219 (2010).

12. C. Vozzi et al., Phys. Rev. Lett. 95, 153902 (2005).

13. I. V. Schweigert, S. Mukamel, Phys. Rev. Lett. 99 , 163001 (2007)

14. E. Seres, J. Seres, C. Spielmann, Appl. Phys. Lett. 89 181919 (2006)

15. S. Mathias et al., Proc. Natl. Acad. Sci. U.S.A. 109 $4792(2012)$
16. K. C. Kulander, K. J. Schafer, ]. L. Krause, in Super-Intense Laser-Atom Physics, B. Piraux, A. L'Huillier, K. Rzazewski, Eds. (Plenum Press, New York, 1993), vol. 316, pp. 95-110.

17. M. Lewenstein, P. Balcou, M. Y. Ivanov, A. L'Huillier, P. B. Corkum, Phys. Rev. A 49, 2117 (1994).

18. J. A. Armstrong, N. Bloembergen, ]. Ducuing, P. S. Pershan, Phys. Rev. 127, 1918 (1962).

19. C. G. Durfee et al., Phys. Rev. Lett. 83, 2187 (1999).

20. E. Constant et al., Phys. Rev. Lett. 82, 1668 (1999).

21. B. Shan, Z. H. Chang, Phys. Rev. A 65, 011804 (2002).

22. J. Tate et al., Phys. Rev. Lett. 98, 013901 (2007).

23. M. V. Frolov, N. L. Manakov, A. F. Starace, Phys. Rev. Lett. 100, 173001 (2008)

24. J. A. Pérez-Hernández, L. Roso, L. Plaja, Opt. Express 17, 9891 (2009).

25. T. Popmintchev et al., Proc. Natl. Acad. Sci. U.S.A. 106, 10516 (2009).

26. M. C. Chen et al., Phys. Rev. Lett. 105, 173901 (2010).

27. T. Popmintchev, M.-C. Chen, P. Arpin, M. M. Murnane, H. C. Kapteyn, Nat. Photonics 4, 822 (2010).

28. G. Andriukaitis et al., Opt. Lett. 36, 2755 (2011).

29. Methods are detailed in the supplementary materials available on Science Online.

30. C. La-O-Vorakiat et al., Phys. Rev. Lett. 103, 257402 (2009).

31. C. La-O-Vorakiat et al., Phys. Rev. X 2, 011005 (2012).

32. M. V. Ammosov, N. B. Delone, V. P. Krainov, Sov. Phys. JETP 64, 1191 (1986).

33. B. Shim, S. E. Schrauth, A. L. Gaeta, Opt. Express 19, 9118 (2011).

34. M. Mlejnek, E. Wright, ]. V. Moloney, Phys. Rev. E 58, 4903 (1998).
35. M. D. Seaberg et al., Opt. Express 19, 22470 (2011). 36. C. G. Schroer et al., Phys. Rev. Lett. 101, 090801 (2008).

37. C. Hernández-García et al., Phys. Rev. A 82, 033432 (2010).

38. I. Thomann et al., Opt. Express 17, 4611 (2009).

Acknowledgments: The experimental work was funded by a National Security Science and Engineering Faculty Fellowship, and the NSF Center for EUV Science and Technology. A.G., A.J.-B., M.M.M., H.C.K. and A. Becker acknowledge support for theory from the U.S. Air Force Office of Scientific Research (grant no. FA9550-10-1-0561)

A. Baltuška acknowledges support from Austrian Science Fund (FWF, grant no. U33-16) and the Austrian Research Promotion Agency (FFG, Project 820831 UPLIT); and C.H.-G. and L.P. acknowledge support from Junta de Castilla y León, Spanish MINECO (CSD2007-00013 and FIS2009-09522), and from Centro de Láseres Pulsados, CLPU. T.P., M.-C.C., A. Bahabad M.M.M. and H.C.K. have filed for a patent on "Method for phase-matched generation of coherent soft and hard X-rays using IR lasers," U.S. patent application 61171783 (2008)

\section{Supplementary Materials}

www.sciencemag.org/cgi/content/full/336/6086/1287/DC1 Materials and Methods

Supplementary Text

Figs. $\mathrm{S} 1$ to $\mathrm{S} 5$

References (39-46)

28 December 2011; accepted 12 April 2012 10.1126/science. 1218497

\section{The Heliosphere's Interstellar Interaction: No Bow Shock}

\section{J. McComas, ${ }^{1,2 *}$ D. Alexashov, ${ }^{3}$ M. Bzowski, ${ }^{4}$ H. Fahr, ${ }^{5}$ ]. Heerikhuisen, ${ }^{6}$ V. Izmodenov, ${ }^{3}$ M. A. Lee, ${ }^{7}$ E. Möbius, ${ }^{7,8}$ N. Pogorelov, ${ }^{6}$ N. A. Schwadron, ${ }^{7}$ G. P. Zank $^{6}$}

As the Sun moves through the local interstellar medium, its supersonic, ionized solar wind carves out a cavity called the heliosphere. Recent observations from the Interstellar Boundary Explorer (IBEX) spacecraft show that the relative motion of the Sun with respect to the interstellar medium is slower and in a somewhat different direction than previously thought. Here, we provide combined consensus values for this velocity vector and show that they have important implications for the global interstellar interaction. In particular, the velocity is almost certainly slower than the fast magnetosonic speed, with no bow shock forming ahead of the heliosphere, as was widely expected in the past.

$\mathrm{T}$ he ionized solar wind flows continuously outward at speeds of $\sim 300$ to $800 \mathrm{~km} \mathrm{~s}^{-1}$, incorporating interstellar neutral atoms that flow into the heliosphere and are ionized to become pickup ions (PUIs). Because the solar wind and surrounding local interstellar medium (LISM) are both magnetized plasmas and cannot penetrate each other, the solar wind inflates a bubble in the LISM called the heliosphere. Inside its boundary, the heliopause, there is a ter-

\footnotetext{
${ }^{1}$ Southwest Research Institute, San Antonio, TX 78228, USA ${ }^{2}$ University of Texas at San Antonio, San Antonio, TX 78249, USA. ${ }^{3}$ Moscow State University, Space Research Institute (IKI) and Institute for Problems in Mechanics, Russian Academy of Sciences, Moscow, Russia. ${ }^{4}$ Space Research Centre of the Polish Academy of Sciences, Warsaw, Poland. ${ }^{5}$ University of Bonn, Bonn, Germany. 'University of Alabama, Huntsville, AL 35805 , USA. ${ }^{7}$ Space Science Center, University of New Hampshire, Durham, NH 03824, USA. ${ }^{8}$ Space Science and Applications, Los Alamos National Laboratory, Los Alamos, NM 87545, USA

*To whom correspondence should be addressed. E-mail: dmccomas@swri.edu
}

mination shock (TS), where the solar wind and PUIs are compressed and heated. Because the heliosphere moves with respect to the LISM, the dynamic pressure plays an important role in shaping the heliosphere, with a compressed "nose" on the upwind side and a downwind "tail" (1). Since
Parker's original work (1), there have been numerous theoretical enhancements, including the addition of an upstream bow shock (BS) (2) that was debated early on (3) but is now widely accepted [for example, see (4-7) and references therein].

NASA's Interstellar Boundary Explorer (IBEX) (8) measures neutral atoms, which move freely across magnetic fields; some of these atoms penetrate from the LISM to $1 \mathrm{AU}$ (astronomical unit: Sun-to-Earth distance), where IBEX detects them. IBEX was primarily designed to measure energetic neutral atoms (ENAs) generated by charge exchange between the solar wind and PUIs (4-7) with interstellar neutrals. These observations led to the detection of an enhanced "ribbon" of ENA emissions nearly encircling the heliosphere, apparently ordered by the external LISM magnetic field and not predicted by any prior model or theory $(9-11)$.

The IBEX-Lo instrument (12) was also designed to measure the neutral interstellar gas

Table 1. Interstellar flow parameters in ecliptic (j2000) and galactic coordinates.

Parameter Value and $1 \sigma$ uncertainty Bounding range

along Eqs. 1 to 3

\begin{tabular}{|c|c|c|}
\hline Speed $\left(V_{I S M_{\infty}}\right)$ & $23.2 \pm 0.3 \mathrm{~km} \mathrm{~s}^{-1}$ & $21.3 \mathrm{~km} \mathrm{~s}^{-1}, 82.0^{\circ},-4.84^{\circ}, 5000 \mathrm{~K}$ \\
\hline Ecliptic longitude $\left(\lambda_{\mathrm{ISM} \infty}\right)$ & $79.00^{\circ} \pm 0.47^{\circ}$ & to \\
\hline Ecliptic latitude $\left(\beta_{\mathrm{ISM}}\right)$ & $-4.98^{\circ} \pm 0.21^{\circ}$ & $25.7 \mathrm{~km} \mathrm{~s}^{-1}, 75.5^{\circ},-5.14^{\circ}, 8300 \mathrm{~K}$ \\
\hline Interstellar He temp. $\left(T_{\text {He⿻ }}\right)$ & $6300 \pm 390 \mathrm{~K}$ & \\
\hline Speed $\left(V_{\mid S M_{\infty}}\right)$ & $23.2 \pm 0.3 \mathrm{~km} \mathrm{~s}^{-1}$ & $21.3 \mathrm{~km} \mathrm{~s}^{-1}, 186.62^{\circ},-9.36^{\circ}, 5000 \mathrm{~K}$ \\
\hline Galactic longitude $\left(I_{I S M_{\infty}}\right)$ & $185.25^{\circ} \pm 0.24^{\circ}$ & to \\
\hline Galactic latitude $\left(b_{1 \mathrm{SM} \infty}\right)$ & $-12.03^{\circ} \pm 0.51^{\circ}$ & $25.7 \mathrm{~km} \mathrm{~s}^{-1}, 183.77^{\circ},-15.22^{\circ}, 8300 \mathrm{~K}$ \\
\hline Interstellar He temp. $\left(T_{\text {Heо }}\right)$ & $6300 \pm 390 \mathrm{~K}$ & \\
\hline
\end{tabular}




\section{Science}

\section{Bright Coherent Ultrahigh Harmonics in the keV X-ray Regime from Mid-Infrared Femtosecond Lasers}

Tenio Popmintchev, Ming-Chang Chen, Dimitar Popmintchev, Paul Arpin, Susannah Brown, Skirmantas Alisauskas, Giedrius Andriukaitis, Tadas Balciunas, Oliver D. Mücke, Audrius Pugzlys, Andrius Baltuska, Bonggu Shim, Samuel E. Schrauth, Alexander Gaeta, Carlos Hernández-García, Luis Plaja, Andreas Becker, Agnieszka Jaron-Becker, Margaret M. Murnane and Henry C. Kapteyn

Science 336 (6086), 1287-1291.

DOI: $10.1126 /$ science.1218497

\section{From Long to Short}

When you play a string instrument, you raise the frequency, or pitch, of the note by shortening the vibrating portion of the string: Drop the length in half, and you hear a harmonic at double the frequency. It is possible to do essentially the same thing with light waves by using selective excitation and relaxation processes of the electrons in crystals or high-pressure gases through which the beam of light is directed to produce light harmonics. Over the past decade, researchers have been optimizing the conversion of red light to the far edge of the ultraviolet, which corresponds to tens of harmonics. Popmintchev et al. (p. 1287) now show that mid-infrared light can undergo a process in high-pressure gas to generate ultrahigh harmonics up to orders greater than 5000 in the $\mathrm{x}$-ray regime.

ARTICLE TOOLS

SUPPLEMENTARY

MATERIALS

REFERENCES

PERMISSIONS http://science.sciencemag.org/content/336/6086/1287

http://science.sciencemag.org/content/suppl/2012/06/07/336.6086.1287.DC1

This article cites 43 articles, 5 of which you can access for free http://science.sciencemag.org/content/336/6086/1287\#BIBL

http://www.sciencemag.org/help/reprints-and-permissions

Use of this article is subject to the Terms of Service

Science (print ISSN 0036-8075; online ISSN 1095-9203) is published by the American Association for the Advancement of Science, 1200 New York Avenue NW, Washington, DC 20005. The title Science is a registered trademark of AAAS.

Copyright @ 2012, American Association for the Advancement of Science 